\title{
Correction of postpneumonectomy syndrome with tridimensional carbon fiber-printed implant
}

\author{
Bin Wang, MD, Sichuang Tan, MD, and Fenglei Yu, MD, Changsha, China
}

\footnotetext{
From the Department of Thoracic Surgery, The Second Xiangya Hospital, Central South University, Changsha, China.

This research obtained technology support from Changsha Kangya Biotech Company.

Disclosures: Authors have nothing to disclose with regard to commercial support.

Received for publication Sept 26, 2017; revisions received Nov 9, 2017; accepted for publication Nov 16, 2017; available ahead of print Feb 2, 2018.

Address for reprints: Fenglei Yu, MD, Department of Thoracic Surgery, The Second Xiangya Hospital, Central

South University, No. 139 Renmin Rd, Changsha, Hunan 410011, China (E-mail: yufenglei@csu.edu.cn). J Thorac Cardiovasc Surg 2018;155:e135-7

$0022-5223 / \$ 36.00$

Copyright $\odot 2018$ by The American Association for Thoracic Surgery

https://doi.org/10.1016/j.jtcvs.2017.11.081
}

$\square$ Video clip is available online.

An 18-year-old woman presented with a more than 6-month history of recurrent dyspnea, wheezing, recurring right bronchopneumonia, and tachycardia after left pneumonectomy 2 years previously for an adenoid cystic carcinoma

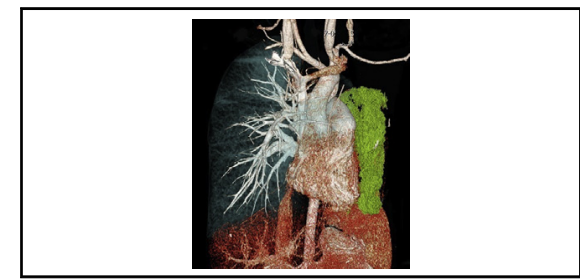

3D carbon fiber-printed implant for PPS surgery.

\section{Central Message}

Conventional implanted prostheses for PPS therapy are easily deformed and liable to shift out of position. 3D carbon fiber-printed implants may be a better option for PPS surgery.

See Editorial Commentary page e139.

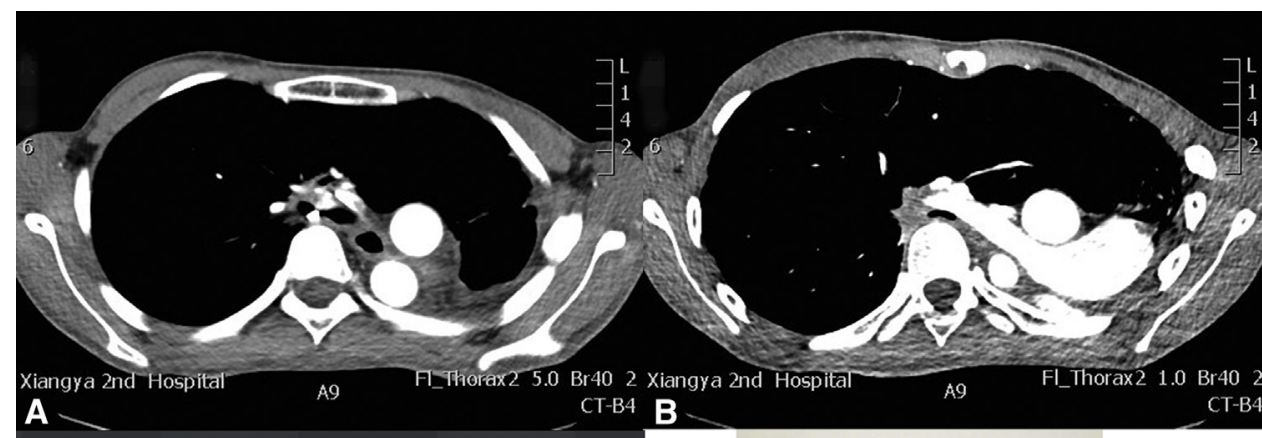

of the left main-stem bronchus. Thoracic computed tomography revealed a shift of mediastinal contents and parts of the right lung into the left hemithorax (Figure 1, $A$ and $B$ ). The heart abutted the left lateral rib cage, and the vena
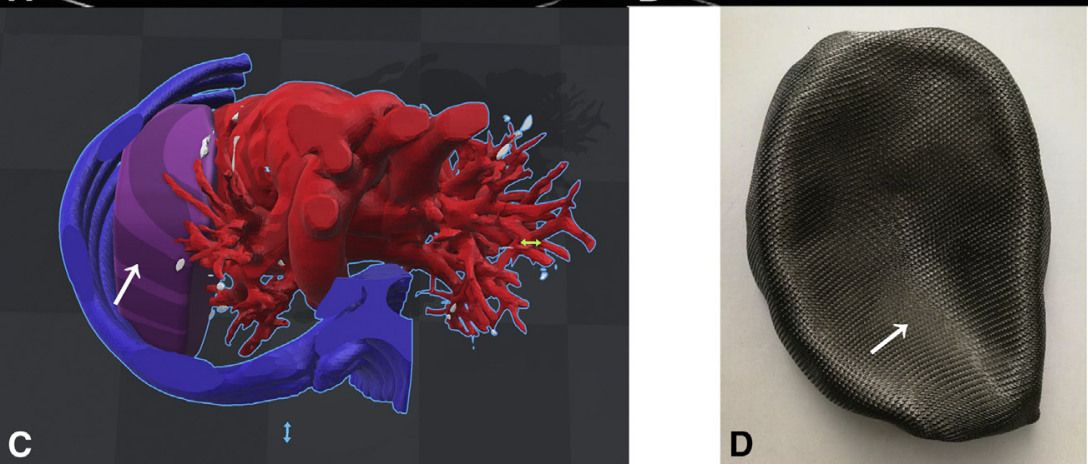

FIGURE 1. A and B, Initial computed tomography scan of the thorax. Shift of mediastinal contents into the left hemithorax (A). The lower trachea and the intermediate bronchus are compressed by the vertebral column and right pulmonary artery (B). C, 3D electron model of the thoracic structure created by MIMICS software (Materialise Magics; Materialise Software, Leuven, Belgium) with computed tomography data. White arrow indicates the simulated implant in the 3D model. D, Custom-made implant created with the carbon fiber 3D sintering printing technique. The 3D printed adjoining plane fits perfectly in the mediastinum. 

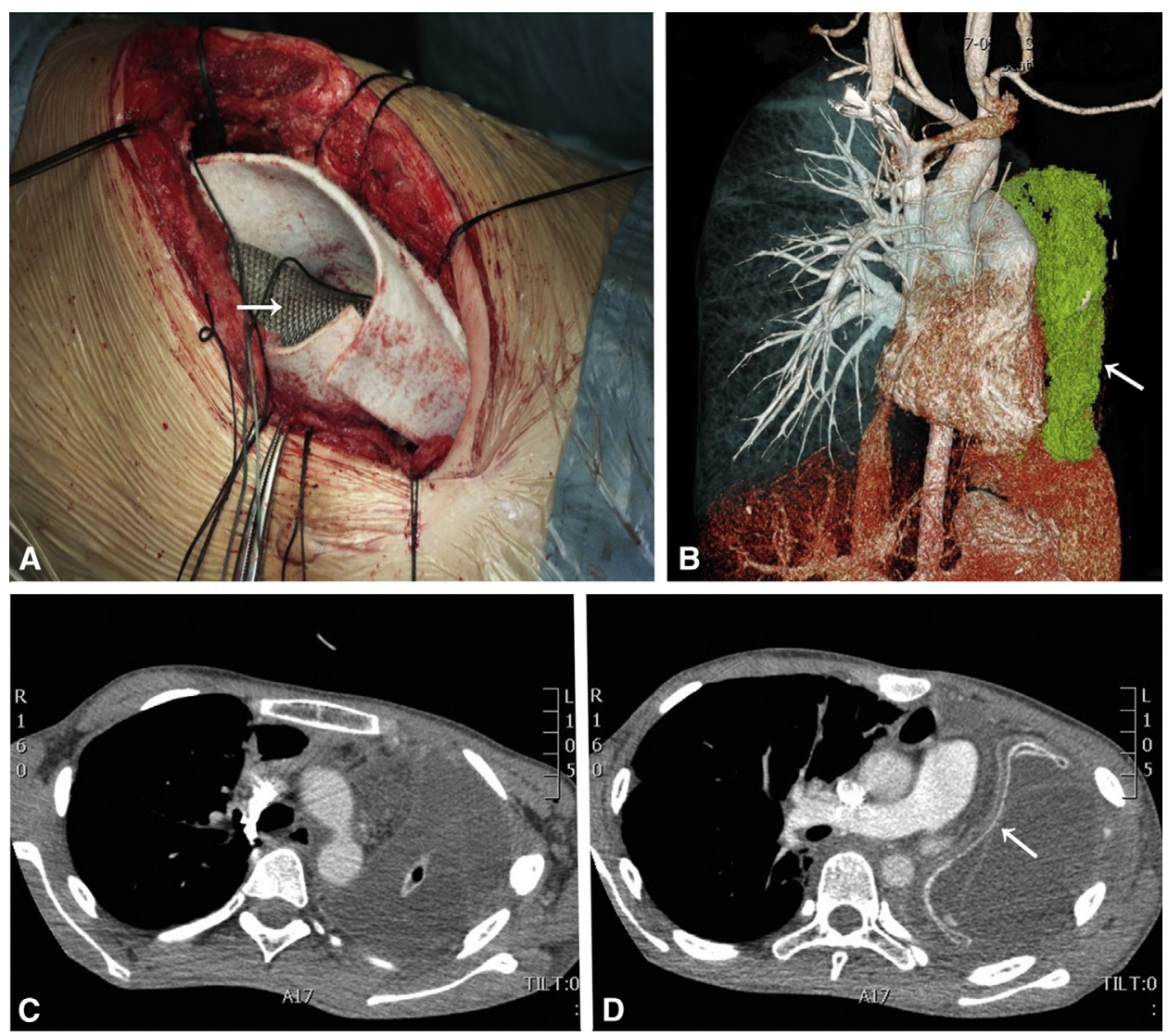

FIGURE 2. A, The custom prosthesis implanted into the left hemithorax cushioned by Teflon patches. B and D, Computed tomography scan of the thorax 12 months after surgery. Arrow indicates the custom implant. B, 3D reconstruction of the intrathoracic structure. C, Normal positioning of the mediastinal contents and right lung. The compressed lower trachea has returned to its normal anatomic position. $\mathrm{D}$, The compressed intermediate bronchus has been relieved.

cava and pulmonary vessels were compressed (Figure 1,B). The most serious effect was that the narrow lower trachea and intermediate bronchus had been compressed by the vertebral column and adjacent viscera (Figure 1, $A$ and $B)$. The preoperative minimal minor axes of the lower trachea and the intermediate bronchus were $2.9 \mathrm{~mm}$ and $2.7 \mathrm{~mm}$, respectively. Bronchoscopy confirmed dynamic severe narrowing of the bronchus. The clinical signs and imaging indicated a diagnosis of postpneumonectomy syndrome (PPS).

To shift the mediastinal contents, especially the trachea, back into their native positions and relieve the compression on the bronchus, a tridimensional (3D) carbon fiber-printed prosthesis was proposed and approved by a multidisciplinary committee. First, a 3D electron model of the thoracic structure, including the organs and ribcage, was created by MIMICS software (Materialise Magics; Materialise Software, Leuven, Belgium) with computed tomography data analysis. The mediastinal shifts and structural improvement of the implant were then performed in the 3D electron model (Figure 1, C). A custom implant was manufactured with the carbon fiber 3D sintering printing technique (Yakang Biotech, Changsha, China) (Figure 1, D). After surgically removing the chest adhesions, the prosthesis was placed in the left hemithorax, cushioned by Teflon patches (Figure 2, A). The mediastinal anatomy shifted back into a normal position (Video 1). Intraoperative bronchoscopy showed a patent right bronchus without narrowing.

The PPS symptoms completely disappeared after surgery, and the patient was discharged 2 weeks later. She remained asymptomatic during 1-year follow-up. Radiographic results showed that the carbon fiber prosthesis had allowed significant reduction of the mediastinal contents and right lung (Figure 2, $B$ and $D$ ), compression of the lower trachea, and relief of intermediate bronchus (Figure 2, $C$ and $D$ ).

The institutional review board of The Second Xiangya Hospital approved the study. The patient gave informed written consent to report her case.

\section{DISCUSSION}

PPS is a rare complication of pneumonectomy that is more commonly seen in adolescent patients. ${ }^{1}$ The preferred treatment strategy for patients with PPS is to shift the 


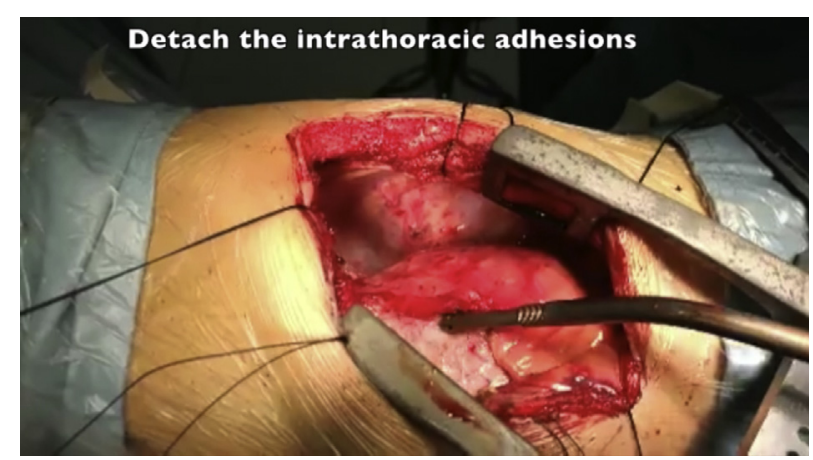

VIDEO 1. The operative procedure of PPS correction with 3D carbon fiber-printed implant. Video available at: http://www.jtcvsonline.org/article/ S0022-5223(17)32841-6/fulltext.

displaced mediastinal contents back into their normal positions with a prostheses. Conventional prosthetic implants for PPS therapy are silicone breast implants, ${ }^{2}$ saline breast implants, and tissue expanders. ${ }^{3,4}$ Because of their lack of rigidity, however, these traditional, flexible material prostheses are easily deformed and liable to shift out of position. It is difficult to maintain stable or reliable support in the mediastinum. The extrusion of contents also may lead to implant failure. ${ }^{3}$ Conventional rigid implants, although not easily deformed, are difficult to fit in the thoracic cavity during surgery. In addition, most of the implants are too heavy. The development of the 3D printing technic and individual therapy have provided a novel solution for PPS surgery: a custom-made 3D printed prosthesis. With better rigidity and strength, the custom 3D carbon fiber-printed prosthesis maintains its form within the chest. With a customized 3D printed adjoining plane, the prosthesis perfectly fits the mediastinal anatomy (especially the ventricular wall) (Figures 1,D, and 2,B). Although the $3 \mathrm{D}$ printed implant's adjoining plane makes the prosthesis perfectly fit the mediastinum, we covered the carbon fiber prosthesis with Teflon patches to offer better cushioning. We also applied fixed Teflon patches to the chest wall before implantation, which avoided a shift of the prosthesis after the operation. The prosthesis did not cause any subjective discomfort or show any signs of erosion. We will continue to follow the patient to confirm the long-term effect. The relatively low density of the material $\left(1.5 \mathrm{~g} / \mathrm{cm}^{3}\right)$ and hollow structure provided a prosthesis for our patient with a weight of $48.6 \mathrm{~g}$, and yet completely relieved the burden of the displaced mediastinal contents. Furthermore, the carbon fiber implant will not interfere with the postoperative imaging around the implants, block the radiation therapy, or decrease the computational accuracy of the therapeutic dose, which may be caused by a metal prosthesis.

\section{CONCLUSIONS}

We report the first case of PPS surgery with a customized 3D carbon fiber-printed prosthesis. This is a novel individual treatment for PPS with satisfactory results.

The authors thank Nancy Schatken, BS, MT(ASCP), from Liwen Bianji, Edanz Group China, for editing the English text of a draft of this article.

\section{References}

1. Valji AM, Maziak DE, Shamji FM, Matzinger FR. Postpneumonectomy syndrome: recognition and management. Chest. 1998;114:1766-9.

2. Wasserman K, Jamplis RW, Lash H, Brown HV, Cleary MG, Lafair J. Post-pneumonectomy syndrome. Surgical correction using Silastic implants. Chest. 1979; 75:78-81.

3. McRae MC, Detterbeck FC, Narayan D. Correction of postpneumonectomy syndrome using a custom implant. BMJ Case Rep. 2011;2011.

4. Lloyd MS, Wallis C, Muthialu N, Elliott M, Bulstrode NW. Treatment of postpneumonectomy syndrome with tissue expanders: the Great Ormond Street Hospital experience. J Plast Reconstr Aesthet Surg. 2014;67:725-8. 